\title{
Pleural infection: a closer look at the pathogenesis, microbiology and role of antibiotics
}

\author{
Abstract \\ BACKGROUND: Pleural infection is a condition that continues to pose a significant challenge to \\ respiratory physicians. We hypothesise that the main barriers to progress include a limited \\ understanding of the pathogenesis, microbiology and role of antibiotics in the pleural space.
}

DATA SOURCE: PubMed was searched for articles related to adult pleural infection using the terms 'pleural infection', 'empyema' and 'parapneumonic'. The search focused on developments in the last ten years, with any older citations only used to display context or lack of progress. Tuberculous pleural infection was excluded.

RESULTS: We summarise our latest understanding of the pathogenesis of pleural infection, including recent advances in diagnostics and biomarkers. We discuss our understanding of the pleural microbiome, which differs inherently to that of the lung, and rationalise the current use of antibiotics in treating this condition.

\section{Introduction}

Pleural infection is a serious illness and continues to be associated with high morbidity and mortality[1,2]. It is extremely concerning that the last decade has seen a doubling of incidence amongst all age groups with a shift in age distribution towards the elderly consistently reported[35]. Outcomes remain poor with a 1 year mortality at $20 \%$, rising up to $30 \%$ in the elderly, whilst 1 in 5 patients will fail medical treatment and require surgery. This high morbidity comes with a significant economic burden associated with prolonged hospital stays, averaging 13 days [6]and United States data estimating annual costs at $\$ 500$ million[7]. Progress is limited by features of the pathogenesis that we do not fully understand, such as the role of biofilm formation. There is also a paucity of data on the causative microbiota and their biology in the pleura, as well as the pharmacokinetics of antibiotics in the infected pleural space. The pathobiology of pleural infection is highly intricate, involving many cells and mediators with complex interactions. The conventionally named 'parapneumonic' effusion, suggesting that the condition was an extension of a severe pneumonia spectrum, is certainly an oversimplification.

\section{Pneumonia vs Pleural Infection}




\subsection{The pathogenesis of pneumonia}

Pneumonia can be defined as an infectious process resulting from the invasion and overgrowth of microorganisms in lung parenchyma, breakdown of host defences and provocation of intra-alveolar exudates[8]. The causes can be divided into intrinsic and extrinsic factors. Extrinsic factors include exposure to a causative agent, pulmonary irritants or direct injury. Intrinsic factors are related to the host relating to loss of upper airway reflexes, which allows aspiration of contents and microorganisms from the upper airways into the lung.

Underlying disease, loss of mechanical respiratory defences with the use of sedatives, tracheal intubation, and antibiotic treatment are all determinant factors for change in the normal flora of the upper respiratory tract. Once these bacteria find their way to down to the lung parenchyma , a combination of factors may lead to bacterial pneumonia. These include virulence of infecting organism, status of local defences and overall health of the patient. Susceptibility to infection is usually increased in patients of advanced age, or those with chronic disease, due to impairment of the immune response. Dysfunction of defence mechanisms, such as is seen in smokers, patients with chronic obstructive pulmonary disease and tumours, can also lead to greater susceptibility.

\subsection{The evolution of the parapneumonic effusion}

\subsubsection{The exudative phase}

The interval between aspiration of organisms and the development of pneumonia varies from a few days up to one week. Pneumonia typically begins in dependent lobes at the periphery of the lung, and if untreated, spreads centripetally towards the hilum. If left untreated for the subsequent 2-5 days, a 'simple' parapneumonic effusion will develop and this is known as the "exudative" phase[9]. The development of the initial effusion is due to increased permeability of the pleural membranes in response to inflammation in the underlying lung parenchyma, which is thought to result in transfer of the interstitial fluid across the visceral pleura. Indirect clinical evidence suggests that pleural inflammation alone is insufficient to result in significant pleural fluid, as many patients complain of pleuritic chest pain during a pneumonic illness, yet only a minority develop a radiologically detectable pleural effusion[10].

Locally increased capillary vascular permeability along with activation of immune processes, such as neutrophil migration, leads to fluid movement into the pleural space. Pro-inflammatory cytokines including interleukin (IL)-6, IL-8 and tumour necrosis factor alpha (TNF-alpha) produce changes in the the anatomical shape of pleural mesothelial cells creating intercellular 'gaps', which further enhance permeability and allow accumulation of additional fluid[10-13]. During this initial exudative phase, the fluid will have a normal glucose level and $\mathrm{pH}>7.2$, with no detectable bacteria, and hence no biochemical or microbiological evidence of bacterial invasion. According to previous series[14], at this stage, this 'simple' parapneumonic effusion will usually spontaneously resolve with antibiotic therapy for the underlying pneumonia. 


\subsubsection{The fibrinopurulent phase}

If inflammation persists within the lung parenchyma, secondary bacterial invasion of the pleural space can occur over the subsequent 5-10 days, with profound pathological effects on normal pleural physiology. The exudative phase will begin to transition into the fibrinopurulent stage. This stage is characterised by rapid depression of the normally high levels of fibrinolytic activity in the pleural space. Titres of specific inhibitors of fibrinolytic activity, namely tissue plasminogen activator inhibitor (PAI) 1 and 2 begin to rise[15]. Levels of PAI-1 and PAI- 2 as well as mediators, such as TNFalpha, are directly released from pleural mesothelial cells and are particularly increased in infected pleural fluid compared with malignancy and other causes[15,16]. Naturally, this reduced fibrinolytic activity leads to fibrin deposition over the visceral and parietal pleura with division of the pleural space by fibrinous septae, producing fluid loculations and pleural adhesions. What is interesting is that although effusions of any cause can become loculated, depression of the fibrinolytic system as evidenced by elevated PAI levels and reduced tissue plasminogen activator (tPA) levels, has only been observed in pleural infection[15,16]. Previous studies have also reported that the significantly elevated PAI-1 levels seen at this stage also correlate with residual pleural thickening (RPT)[17], and may be implicated in treatment failures and poor outcomes.

This fibrinopurulent phase is characterised by bacterial metabolism and neutrophil phagocytic activity leading to increased lactic acid production and hence the biochemical hallmarks of early pleural infection - a fall in pleural fluid $\mathrm{pH}$ and glucose[18,19]. As neutrophilia in the pleural space rises, lactate dehydrogenase is released and this is responsible for the typically high levels seen in infected pleural fluid. The term 'complicated' parapneumonic effusion correlates with the early fibrinopurulent phase and implies that pleural intervention in the form of intercostal or surgical drainage, is likely to be required in order to achieve complete resolution of the pleural effusion. In the event that the early fibrinopurulent phase continues to progress, ultimately the fluid becomes frankly purulent, secondary to bacterial and inflammatory cell death and lysis. This late fibrinopurulent stage correlates to an 'empyema' and is defined as frank pus in the pleural space. Regardless of biochemical and microbiological parameters, this is macroscopic evidence of bacterial and inflammatory cell death, requiring immediate drainage.

\subsubsection{The organising phase}

As pleural infection evolves through the exudative and even into the late fibrinopurulent phase, the lung is typically still expandable. A further 10-21 days of suboptimal treatment, will see it entering the final stage known as the 'organising' stage. This is characterised by proliferation of fibroblasts and pleural scarring, where there may be evidence of lung entrapment due to visceral pleural fibrosis, which can result in impaired lung function. Animal model data suggests that this process is driven by mediators such as platelet-derived growth factor[20] and transforming growth factor beta[21]. There is interesting evidence that these mediators may provide a potential therapeutic target through administration of anti-TGF-beta antibodies during pleural infection resulting in significantly less pleural thickening in a well-established animal model of empyema[22]. The establishment of this organised phase is also important clinically as it potentially marks the point 
where drainage of an infected pleural collection with a chest tube, even when supplemented by fibrinolytics, is likely to fail. This is because tissue plasminogen activator (tPA) and deoxyribonuclease (DNAse) may lyse fibrin but not collagenous fibrous tissue, and clinicians have assumed that this is the time when the patient is likely to require decortication in order to recover. The counter to that is there is marked inter-patient variation in the timescale in which this occurs, as approximately $50 \%$ of patients will not yet have developed collagenous fibrous pleural scarring even three weeks after pleural infection onset[23]. This is of particular importance, particularly in the elderly patient with comorbidity who is likely to be a less favourable surgical candidate, and implies that chest tube drainage or minimally invasive thoracoscopic surgery may still be effective even when there is a long history of illness at presentation. Interestingly, the subsequent clinical course after the organising phase is entered is also variable, with some patients undergoing spontaneous resolution of pleural thickening at 12 weeks[24], while others develop chronic sepsis and lung function deficits[25].

\subsection{Is pneumonia always involved?}

About $60 \%$ of cases of pleural infection are related to a primary pneumonic process, therefore risk factors for pleural infection are assumed to be similar to those for pneumonia[10]. The presence of an effusion in patients with pneumonia is known to be associated with a 3-6 fold increase mortality[26-28]. It is important to however highlight that empyema may be secondary to a nonpneumonic process. Indeed, pleural infection has been shown to develop spontaneously without underlying lung consolidation and approximately $30 \%$ of patients with pleural infection have no evidence of pneumonia on CT imaging[29].

A significant portion of these are thought to develop from haematogenous spread of bacteraemia and some groups of patients may be more predisposed to this, such as patients with liver cirrhosis in the presence of a hepatic hydrothorax, so called 'spontaneous bacterial empyema' (SBEM). It is important to note that in cases of true SBEM, treatment comprises of intravenous antibiotics and albumin and generally, thoracostomy tubes should be avoided unless the fluid is grossly purulent. The rationale for this being that chest tube drainage may result in life threatening fluid depletion, protein loss and electrolyte imbalance[30]. Other possible routes for development of pleural infection include translocation through visceral pleural defects or fistulae in the context of lung cancer, post radiotherapy or postoperatively. Further stipulated mechanisms involve penetrating injury across the parietal pleural in the context of trauma or chest tube insertion, spread from the mediastinum in cases of oesophageal rupture, as well as transdiaphragmatic spread in the context of intraabdominal infection and alcoholic cirrhosis[29,31,32].

Independent risk factors for development of empyema include diabetes, immunosuppression, gastro-oesophageal reflux disease, alcohol, intravenous drug abuse, aspiration and poor oral hygiene[1]. In 2009, Chalmers et al prospectively identified seven clinical factors that independently predict the development of a complicated parapneumonic effusion or empyema. Whilst these were not independently validated, they include albumin $<30$, platelet count $>400, C$-reactive protein $>100$ and a history of alcohol abuse or intravenous drug use. Unlike pneumonia, a history of COPD was actually associated with a decreased risk[33]. 


\section{Microbiology}

A large number of micro-organisms are capable of infecting the pleural space. The pattern of microbiology varies widely according to different factors including the source (haematogeneous spread, parapneumonic or iatrogenic following thoracic surgery) and setting of infection (hospital versus community acquired), the age group, and the geographic location where the infection takes place. All of these factors together with the aptitude of particular organisms than others to inhabit the pleural space determine the common types of organisms that cause infection.

\subsection{Community vs Hospital}

In a recent review, data from three large studies on pleural infection reporting results of pleural fluid culture from 974 patients [34-36] were combined to show the most common organisms responsible for community-acquired (CA) and hospital-acquired (HA)pleural infections.[37] The most common CA aerobic organisms were the viridans Streptococci (a group comprising Streptcoccus (Strept.) milleri and other oral commensals from the genus) (25\%), Strept. pneumoniae (23.8\%), Staphyloccocus(Staph.) aureus (14.5\%) and Enterobacteriacae (7.5\%). The most common HA aerobic organisms were Staph. aureus (45\%), Enterobacetriacae (19.6\%), viridans Streptococci (9\%) and Pseudomonas spp (6.5\%). Anaerobic bacteria were found in $18 \%$ of the CA infections and in $11 \%$ of HA infections.

The difference in the organisms that cause CA as opposed to HA pleural infection is, at least in part, related to the different risk factors of the two patient groups. Impaired conscious levels and higher risk of aspiration is far more common in hospitalized patients which explain the higher incidence of gram-negative gut bacteria. [38] On the other hand, poor dental hygiene is well characterized as a risk factor for CA pleural infection[38,39] and explains the prominent role played by the microaerophilic oral Streptococci (including the viridans groups and pneumococci).

Another important difference between CA and HA pleural infections is the rate of antibiotic resistance, which is a known risk factor for any HA infection. Methicillin-resistant Staph. aureus represented $25.8 \%$ of infections caused by the organism in CA infections while it was reported in $68.8 \%$ of infections caused by Staph aureus. in HA infections.[34-37]

\subsection{Geography}

The Strept. milleri group represents the most common isolates reported in studies from North America, Europe and Australia. [40] Reports from East Asia, and particularly Taiwan and Korea, show higher proportions of Klebsialla pneumonia infection than is seen in reports from other regions both in $\mathrm{CA}[41,42]$ and $\mathrm{HA}[34]$ infections. The cause of this variation is not completely understood, but it may be related to climatic factors such as differences in ambient temperatures and humidity levels. A systematic review is underway to specifically examine the geographical variation of microbiology in bacterial infection worldwide (CRD42017076418).

Among the less known causes of bacterial pleural infection that has a strong geographic pattern is melioidosis. This is an infection endemic to south east Asia and north Australia that is caused by the gram negative bacillus Burkholderia pseudomallei . It typically involves the lung in the acute stage 
and requires relatively long durations of intravenous antibiotics to be eradicated. [43] Pleura infection complicates about $28 \%$ of cases with pulmonary meliodosis. [44]

\subsection{Specific organisms and risk factors}

The Streptococcus genus is composed of 40 species that are commensals or temporary colonisers of the upper or lower $\mathrm{Gl}$ tract. The genus is subclassified into six species groups based on various biochemical, antigenic or chromogenic tests. [45] As previously mentioned in this review, the milleri group as well as pneumococci (member of the 'mitis' group) are among the top causes for pleural infection. Other isolates from the 'salivarius' and 'mutans' groups are also occasionally reported in pleural fluid culture results. These four groups are traditionally termed the oral group or the 'viridans' group. However, beta-haemolytic steprococci, a member of the (non-viridans) pyogenic group are also relatively common isolates. These oropharyngeal microbes are rarely seen in pneumonia, but they are common causes for pleural infection and lung abscesses. [46]

Streptococci commonly co-infect the pleural space with anaerobic bacteria.[38,47] Anaerobic bacteria are important causes for both CA and HA infections. Culturing these organisms is demanding and more technically difficult which could mean that the percentages reported in the above studies could be an underestimate. In a study applying different culture methods dedicated for isolating anaerobic bacteria, these organisms were found in $74 \%$ of samples of infected pleural fluid[47] and co-infection of the pleural space with aerobic and anaerobic bacteria was seen in more than $60 \%$ of the positive samples.[47]

Streptococcus pneumoniae are the most common cause of CA bacterial pneumonia and are associated with pleural effusion in more than one third of the cases, although many of these effusions do not represent true pleural infection.[48] Besides being an important cause for CA pleural infection in adults, the organism is responsible for more than $80 \%$ of pleural infection in the paediatric population.[49] Due to the widespread use of the pneumococcal vaccine, a recent increase of pleural infection by more virulent serotypes (that are not covered by the vaccine) is noticed.[50]

Staphylococcus aureus is a common pathogen in HA pleural infections in a manner similar to what is known about the aetiology of HA infections of other organs of the body. A major problem with Staph. aureus is antibiotic resistance which is seen both in CA and HA pleural infection, though with different proportions.[38]

Another common group of organisms in HA infections are the lower gastro-intestinal or enteric bacteria. Both the Enterobacteriaceae group (including Klebsiella spp.) and Enterococci, which are gram-positive organisms closely related morphologically to streptococci but resides in the lower GI tract[45], are among the common causes of HA infection. Another category of pleural infections that is predominantly caused by enteric bacteria is spontaneous bacterial pleuritis. [38] These are cases with hepatic hydrothorax who develop signs of pleural infection either with or without spontaneous bacterial peritonitis.[51]

Non-bacterial causes of pleural infection are numerous but are mostly reported as case series. Fungal infection of the pleural infection is uncommon, and in more than one third of cases a cause of immune suppression is identified.[52] The most common fungi reported in pleural infection are the Candida spp.[52] Pleural infection secondary to mycobacterium tuberculosis has varying incidences 
according to differences in TB prevalence. For example, TB as a cause of pleural infection was found in $9 \%$ of culture results of pleural fluid in a large UK cohort[35] while that figure was $35 \%$ in an Indian cohort.[53]

\subsection{Outcome}

Various studies found that the outcome of pleural infection is affected by the causative organism and the setting of infection. In a study examining the bacteriology of 164 culture-positive pleural infections, isolates from the Streptococcus genus were the most common, but when specifically looking at patients who required ICU admission, the most common isolate was Klebsiella pneumoniae .[42] In another study involving patients with CA pleural infections, non-Strept. milleri pleural sepsis was associated with longer durations of hospitalization. [54] Analysis of the bacteriology of patients from the MIST1 trial[35] revealed that one-year mortality was significantly worse for patients with infections caused by Staph. aureus or mixed aerobic infection as opposed to infections caused by Strept. milleri or mixed anaerobic infection. The same study looked at the source of infection and its effect on patient outcomes and found that mortality was higher with HA infection (commonly caused by the earlier groups of organisms) in comparison to CA infections (typically caused by the latter group).[35] Another study also found that HA infection was a risk factor for worse 30-day mortality in patients with pleural infection. [34] Moreover, in patients referred for decortication, pleural fluid culture positivity was associated with longer duration of hospital stay. [55] This is likely to be supported by the recent finding that PAI-1 levels are higher in pleural effusions from gram positive bacteria, compared to gram negative and uncomplicated culture negative parapneumonic effusions[56]

\section{Pleural infection diagnostics}

\subsection{The current state of pleural fluid biomarkers}

Current guidelines advocate early diagnostic sampling of pleural fluid using a $\mathrm{pH}<7.2$, glucose $<2.2 \mathrm{mmol} / \mathrm{I}$ and $\mathrm{LDH}>1000 \mathrm{IU} / \mathrm{I}$ to be consistent with a complicated parapneumonic effusion and a diagnosis of pleural infection.

Studies have attempted to identify methods of earlier diagnosis or other means of rationalising pleural fluid drainage. Numerous biomarkers, such as inflammatory cytokines (tumour necrosis factor-alpha/TNF- $\alpha$, interleukin-8/IL-8, and IL-1 $\beta$ ), enzymes (neutrophil elastase, myeloperoxidase/MPO, and metalloproteinases/MMPs), C-reactive protein (CRP), and soluble triggering receptor expressed on myeloid cells (STREM-1), have been evaluated but not proven superior to traditional criteria[57-61]. The utility of serum procalcitonin in the diagnosis of pleural infection was evaluated in a large prospective trial and concluded that this was not superior to serum CRP and WCC for the diagnosis of bacterial pleural infection[62]. In the absence of pleural fluid purulence, Porcel et al suggested that a pleural fluid CRP>100mg/I can aid diagnosis of CPPE and aid decision to drain but this had poor correlation with fluid $\mathrm{pH}$, and was based on retrospective analysis[63].

Other markers such as Interleukin-18 have been found to be linked to the intensity of neutrophilic pleural inflammation in patients with pleural effusions and up-regulated in the pleural space of 
patients with empyema, but clinical applicability remains unclear[64]. Recently, Nature published an interesting prospective study by Wu et al, who conducted comprehensive proteome profiling of pleural fluid from simple and complicated parapneumonic effusions to assess the performance of four novel proteins - BPI, NGAL, AZU1 and calprotectin. Bactericidal permeability-increasing protein protein (BPI), a neutrophil granule protein with antimicrobial activity against bacteria, was found to be superior to to $\mathrm{LDH}$, glucose and $\mathrm{pH}$ in the diagnosis of CPPE with an AUC value of 0.966.

Furthermore, the combination of pleural fluid BPI levels with LDH levels improved the sensitivity to $100 \%$ for identifying CPPE[65]. The authors did not evaluate how using BPI would change management e.g. decision to drain or influence outcomes, so the benefits in a clinical setting are yet to be proven. The finding that BPI levels were twice as high in patients with empyema, makes this an interesting area to explore in the clinical setting against pleural aetiologies that can occasionally mimic pleural infection biochemically, such as MPE and inflammatory pleuritis, when the clinical context is unclear and diagnosis cannot be supported further by negative microbiology.

\subsection{Microbiology: Pleural fluid}

One of the ongoing challenges with treating this condition has been our limited ability to identify the responsible microorganism. This is likely to be due to a combination of prior antibiotic treatment, low bacterial concentration in pleural fluid and possibly causal agents that are difficult to isolate in the laboratory due to stringent requirements. In most cases of pleural infection, the pleural fluid is acidic, hypoxic and lacking in nutrition and we know that bacterial concentration significantly increases in cases with greater fluid purulence[66]. A small study looking into predictors of bacterial 'load', used PCR techniques to analyse 172 pleural fluid samples and found that in the correct clinical context, $\mathrm{pH}$, glucose and LDH values correlated well with bacterial concentration. Interestingly, patient WCC and CRP were of lower significance[67].

Standard pleural fluid cultures are usually positive in approximately $20-40 \%$ of cases[68]. This was shown to be increased to almost $60 \%$ when pleural fluid is inoculated into blood culture bottles. In this prospective controlled study, Menzies et al demonstrated that this technique not only increases the yield, but also has the potential to add further diagnostic value through identifying additional microbiologically relevant organisms when the standard culture is already positive[68]. As well as pleural fluid, the MIST1 study also reflected the importance of sampling blood for culture in blood culture bottles, as $12 \%$ of patients had positive blood cultures with no other positive microbiology[69]. Liquid culture media have been proven to greatly increase the yield in cases of pleural tuberculosis[70], but to the best of the authors' knowledge, this has not been studied in nontuberculous infection.

\subsection{Microbiology: Pleural biopsy}

Whilst pleural tissue biopsy has been widely adopted in endemic areas for suspected tuberculous effusions for a long time, as well as evaluating other causes of pleural disesase, the recently published AUDIO study suggests that this practice may become commonplace for non-tuberculous pleural infection in the near future. Psallidas et al performed ultrasound guided pleural biopsies in patients diagnosed with pleural infection at the same procedure of chest drain insertion and the 
material was sent in normal saline for microbiological examination, alongside standard pleural fluid culture and gram stain, including inoculation of pleural fluid in BACTEC blood culture bottles, and blood cultures. Patients did not have to have evidence of pleural thickening on ultrasound. The results showed a remarkable increase in microbiological yield to $45 \%$ compared to pleural fluid and blood cultures ( $20 \%$ and $10 \%$ respectively)[71]. This was a pilot, feasibility study involving 20 patients but has certainly set the scene for larger clinical studies to evaluate the use of this technique as an additional test to form part of the standard work up of pleural infection.

The results of the AUDIO study do make one wonder why the yield was so much higher from pleural biopsy and how this adds to our understanding of the pathobiology of pleural infection. In their discussion, the authors hypothesized that an improved blood supply and better nutrition may provide a more favourable environment for the bacteria to be located, rather than within the pleural fluid.

Interestingly, pleural biopsy culture positivity was not affected by prior antibiotic administration, and this may simply reflect the limited antibiotic penetration into the pleural space and emphasize the importance of other features of the pathogenesis such as biofilm formation in this condition[71]

\subsection{The role of Nucleic acid amplification testing (NAAT) in pleural infection}

The use of a universal polymerase chain reaction (PCR) of the $16 \mathrm{~S}$ ribosomal ribonucleic acid (rRNA) gene (16S PCR) followed by sequencing has proven to be a reliable complement to conventional culture in a variety of infectious processes including infective endocarditis and bone infections. This was prospectively evaluated in pleural infection in 2 large teaching hospitals in Spain as part of their laboratory routine. The sensitivity of PCR, with respect to standard pleural fluid culture, increased by almost $50 \%$ with no reduction in specificity[72]. This paper, however, discussed several barriers to the general application of $16 \mathrm{~S}$ PCR. These include the high cost, the high level of purity in the reagents used in the extraction process to avoid false negatives, as well as the possibility of obtaining mixed sequence, which would delay diagnosis by several days, defeating the purpose of using such techniques. This in itself presents a further limitation, which is the degree of expertise required to use these technologies.

The AUDIO study investigators also conducted an exploratory phase evaluation of using $16 \mathrm{~S}$ rRNA amplification techniques using pleural fluid samples from the previously published MIST2 study, to design specific primers for microbes. The four specific primer sets tested, demonstrated excellent quantitative PCR (qPCR) based pathogen detection with a specificity (93\%) and sensitivity (89.5\%) for the detection of the four most common pathogens in pleural infection[71]. The potential that the 16S rRNA technique has in identifying a causative organism within a few hours of receiving samples, should really drive further exploration of $\mathrm{qPCR}$ use to improve diagnosis and management. 


\section{Antibiotics in the pleural space}

\subsection{Penetration and concentration in pleural fluid}

In general, it is believed that antibiotic levels in pleural fluid are similar to those in the serum, but most studies in humans involved patients with diseases other than pleural infection[73]. Teixera et al suggested that pleural fluid antibiotic levels are lower than serum levels, due to the decreased permeability of thickened pleura[74]. The acidic environment created by the infected, protein-rich fluid is also likely to affect antibiotic penetrance.[75]. One could counter the reasoning of Teixera et al, by suggesting that under circumstances of acute infection, involving inflammation, vasodilation, oedema and increased membrane permeability, antimicrobial penetration may be increased[76]. Another explanation is that antibiotic penetration actually varies according to the underlying pathophysiology of pleural fluid formation, such that in cases of secondary pleural infection associated with pneumonic inflammation causing greater permeability of the visceral pleura, antibiotic penetration is increased and these patients may respond more favourably.

In the study by Teixera et al, equilibration of serum and pleural fluid levels occurred very rapidly with penicillin and metronidazole and, and along with our knowledge of the associated microbiology, this explains widespread first-line use of these agents. Ceftriaxone, clindamycin and vancomycin followed in that order. Very poor penetration was seen with gentamycin, confirmed by other studies and in keeping with the recommendation against use of aminoglycosides in current guidelines [77]. Using a similar rabbit model, other agents including clindamycin, levofloxacin and moxifloxacin have also been studied. Based on the correlation between pleural fluid and serum antibiotic levels, this would suggest that these may be appropriate alternatives for susceptible organisms[78]. Other commonly used agents including ertapenem and linezolid (particularly in the context of hospital acquired infection and MRSA) were also found to penetrate well into empyemic pleural fluid[79].

Extrapolating conclusions such as this from rabbit data has its limitations due to the difference in visceral pleural thickness (thinner pleura in rabbits), the mechanism of induction of empyema and variation in microbiology between a human and a rabbit. Studies of antibiotic concentrations in human pleural infection are lacking. Chatzika et al conducted a clinical study of intravenous moxifloxacin in 12 patients with pleural infection vs 12 malignant pleural effusion 'controls'. They found it took significantly longer for the antibiotic to reach therapeutic pleural fluid levels in the empyema group, but moxifloxacin appears to exhibit a favourable pharmacokinetic profile regardless of pleural fluid origin[80].

\subsection{Antibiotic choices}

Whilst certainly dated, lacking in humans, and based on ancient regimes, the literature seems to suggest that most antibiotics show good pleural fluid penetration, with the antibiotic exceeding the minimum inhibitory concentration (MIC) for the bacteria for which it would be normally used[73]. The notable exception is gentamicin and current guidelines therefore recommend against the use of 
aminoglycosides in pleural infection[74,77]. Initial selection of agent should always depend on whether the patient is likely to have a community or hospital acquired infection, given our greater understanding in the last decade, of the variation in microbiology (see section 2). This should then be correlated with local hospital policies and antibiotic resistance patterns. In community-acquired infection, treatment with an aminopenicillin will cover the common causative organisms, but a betalactamase inhibitor such as co-amoxiclav or metronidazole should also be given due to the frequent co-existence of penicillin resistant aerobes (including staph aureus) and anaerobic bacteria. Clindamycin, alone, or in combination with ciprofloxacin or a cephalosporin are likely to provide good alternatives for patients with penicillin allergy[77]. In the setting of hospital acquired or postsurgical infection, vancomycin and piperacillin/tazobactam will cover the added risk of MRSA and Pseudomonas. Vancomycin and meropenem may be indicated if there is a history or suspicion of extended spectrum beta-lactamase producing organisms[81]

\subsection{Antibiotic duration}

The duration of antibiotic therapy in pleural infection has been traditionally extrapolated from lung abscess treatment with most experts advocating a minimum of 4 weeks treatment. The switch from intravenous to oral should be guided by cessation of pyrexia, clinical improvement and resolution of inflammatory markers[77,82]. A recent cohort study from the Mayo Clinic studying 91 patients over a 10 year period concluded that three weeks of treatment was generally adequate to prevent treatment failure, with prolongation of the initial intravenous course rather than the oral course associated with fewer cases of treatment failure[83]

\subsection{Intrapleural antibiotics}

Consideration has been given to the role of administering antimicrobial directly into the pleural space, particularly to help circumvent the problem in those classes of antibiotics, which would be considered to have poor pleural penetration when administered intravenously. This appears to be widely practiced in the management of post pneumonectomy empyema (PPE), which has an incidence of approximately $5-10 \%$. Historically, this would have been managed with further aggressive surgical techniques such as rib resection or procedures to obliterate the space such as thoracoplasty, with associated mortality rates of 9-13\%. The evidence base of administering antibiotics in this way is limited to retrospective case series. $\mathrm{Ng}$ et al found that VATS debridement (in patients without bronchopleural fistula) with subsequent intrapleural antimicrobial irrigation (over a mean period of 40 days) resulted in successful management, with no treatment associated morbidity and mortality and no empyema recurrence within the two-year follow up period[84].

It would appear that the exact method of administration, frequency and endpoints to inform treatment discontinuation are highly variable within surgical practice, and this is likely to need further investigation. The case series from $\mathrm{Ng}$ et al used three successive negative cultures as their endpoint. The recent retrospective analysis by Torbic et al in 18 patients, quoting a PPE recurrence 
of $28 \%$, when intrapleural antibiotics were given for 6 days, would suggest that prolonged administration is certainly warranted[85].

The practice of intrapleural antibiotic administration in pleural infection, outside the context of surgery, is much less established. It has been postulated as a potential management option in select cases, however evidence is limited largely to case reports. One such report documents the successful use of intrapleural colomycin in the management of carbapenem-resistant Acinetobacter which resulted in successful resolution following the unsuccessful treatment with systemic colomycin. No data is available regarding the pleural fluid pharmacokinetics of colomycin, but the case reports rapid clinical, radiological and microbiological resolution following the addition of intrapleural antibiotics[86]

With poor penetration of systemic antibiotics into the pleural activity being seen as a major cause of treatment failure, one animal study has examined the use of a novel antibiotic-eluting intrapleural pigtail catheter. This was coated with electrospun nanofibers used for sustained release of bactericidal concentrations of penicillin in the pleural space. Their positive results[87] suggest that this is certainly an interesting area for future research, particularly given the practical difficulties associated with intrapleural administration otherwise, and the prolonged hospital stays involved.

\subsection{The effect of intrapleural enzyme therapy (IET)}

The last decade has seen a major breakthrough in the treatment of pleural infection with the advent of IET. The MIST2 trial found that the intrapleural use of tissue plasminogen activator (t-PA) and DNAse improved the drainage of infected fluid in patients with pleural infection [6]. This is likely to occur principally via a monocyte chemotactic protein (MCP)-1 dependent mechanism[88]. There were also some signals that t-PA and DNase combination therapy may improve the natural history of pleural infection, with a $77 \%$ reduction in referrals to surgery and 6.7 day reduction in hospital stay[6]. The reason that this was so successful is that it probably did more than targeting the fibrinolysis pathway that has been pursued by chest physicians for more than half a century[89]. Given our understanding of the complexity, it would seem logical that clearing an infected pleural space requires more than just breakdown of septations. The presence of extracellular uncoiled DNA liberated from dead leukocytes and other bacterial components is likely to increase viscosity and permit biofilm formation. These components, in combination, are likely to be responsible for hindering drainage. This would also explain why previous studies using other fibrinolytics eg streptokinase[69] were not as successful due to its inability to influence the latter two factors in an in vitro setting[90]. The formation of biofilms, incorporating fibrin as well as DNA, has been described in many common respiratory bacteria, and DNase has been shown to have the ability to interfere with this matrix. This may potentially enhance the effect of antibiotics[91,92]. IET is subject to relatively rapid inactivation by inhibitors including PAI-1, which are present in higher levels in infected pleural effusions. PAI-1 neutralising antibodies have been shown to enhance the effectiveness of low doses of intrapleural fibrinolytic therapy[93]. This has paved the way for a single-chain urokinase plasminogen activator (scuPA), a fibrinolysin that is processed to a PAI-1 resistant form, and is currently in a phase 1 trial for patients with loculated pleural infections $[94,95]$. 


\section{Conclusion}

Pleural infection is an important condition that requires urgent attention, independent of pneumonia. Recent advances continue to unravel new areas of knowledge relating to the pathogenesis, involving the host, the bacteria and the pleural environment. These are vital to future advances in treatment. The microbiology is variable, evidently evolving and likely to influence outcomes in patients, suffering with this condition. Delays in diagnosis, low yields and polymicrobial infections are likely to be ongoing challenges. Further validation of pleural biopsy in larger cohorts is an exciting development and may even extend the spectrum of organisms known to be involved. For now, our knowledge of antibiotic penetration is limited mainly to rabbit models of empyema. The increased incidence in the elderly, with limitations of surgery in this group, means that advances in intrapleural fibrinolytic therapy are going to be key in improving outcomes.

\section{Expert Commentary (500-1000 words)}

There has been an acceleration in studies addressing key management issues in pleural infection in the last 10 years, and much treatment is now evidenced based where before such evidence did not exist, or was based on expert opinion only. However, there remain many areas of clinical unmet need, and key questions on the aetiopathogenesis of pleural infection, which the authors hope will be addressed using clinical and translational studies in the next 5 years.

The key event in so called "parapneumonic" effusion appears to be translocation of bacteria from the infected lung (where this is present) in to pleural fluid, permitting bacterial replication in an immunologically poorly surveyed space. However, the precise mechanism of this key event is poorly understood, and a greater appreciation of the process might permit more targeted therapy which has the potential to prevent this occurring in the first place. Which factors in the bacteria and host permit such translocation is a key area of research in the future, and an assessment of factors differentiating those with "simple" and "complex" effusions is key to further our understanding. One presumes that there will be differences not only in bacterial virulence or invasiveness, but also in the inflammatory milieu of the host which leads to such different phenotypes in clinical practice.

Progression of pleural infection from the exudative to the organising phase is a well rehearsed tenet of the pathogenesis of frank pleural infection, and yet the key steps have not been thoroughly tested in animal models or human studies. A deeper understanding of the fibrinolytic potential of the pleural space, factors associated with fibrin deposition and purulence, and mechanisms of repair and resolution are key to improving treatment in this disease, which is associated with a high morbidity and mortality. Studies to demonstrate that preventing such progression through these phases is associated with clinical benefit are currently lacking, although intrapleural combined enzyme therapy (in the form of the MIST2 regimen) does suggest that manipulating the biological process may hold answers to this question. A key area of uncertainty is currently the meaning and importance of septations detected at ultrasound or loculations on CT, and translational studies to assess this are greatly needed.

Microbiological diagnosis remains challenging, and recent data suggesting higher microbiological yields from pleural biopsy than pleural fluid suggests not only a new clinical diagnostic test, but may 
also provide insights in to the aetiopathogenesis. If bacteria truly "prefer" the pleural lining, this has implications for effective treatment, which must address not just the infected fluid, but achieve resolution of deep seated infection within the thickened and inflammatory pleural lining. It is therefore possible that stripping the infected pleural lining (through surgery or enzymatic debridement) is therefore the optimal treatment, but further studies are now required to address if biofilm formation is a real clinical issue in pleural infection. If established, this has the possibility of radically altering the treatment paradigm, suggesting that early aggressive management may be optimal for these often frail patients.

Finally, effective antibiotic delivery to the pleural space is an area with little evidence, and is likely to be a fertile area for research with the potential to improve outcomes. This aspect involves not only a more thorough assessment of causative bacteria within the infected pleural space, but also issues including achievable effective antibiotic concentrations within the pleura, duration of antibiotic therapy and clinical markers in real practice which suggest treatment success. In the era of antibiotic stewardship, this is particularly important given the increasing prevalence of resistant bacteria.

[1] Maskell NA, Batt S, Hedley EL, et al. The bacteriology of pleural infection by genetic and standard methods and its mortality significance. Am. J. Respir. Crit. Care Med. 2006;174:817823.

[2] Asai N, Suematsu H, Hagihara M, et al. The etiology and bacteriology of healthcare-associated empyema are quite different from those of community-acquired empyema. J. Infect. Chemother. 2017;23:661-667.

[3] Farjah F, Symons RG, Krishnadasan B, et al. Management of pleural space infections: a population-based analysis. J. Thorac. Cardiovasc. Surg. 2007;133:346-351.

[4] Finley C, Clifton J, Fitzgerald JM, et al. Empyema: an increasing concern in Canada. Can. Respir. J. 2008;15:85-89.

[5] Shen HN, Lu CL, Li CY. Epidemiology of pleural infections in Taiwan from 1997 through 2008. Respirology (Carlton, Vic.). 2012;17:1086-1093.

[6] Rahman NM, Maskell NA, West A, et al. Intrapleural Use of Tissue Plasminogen Activator and DNase in Pleural Infection. New England Journal of Medicine. 2011;365:518-526.

[7] Lisboa T, Waterer GW, Lee YCG. Pleural infection: changing bacteriology and its implications. Respirology. 2011;16:598-603.

[8] Alcón A, Fàbregas N, Torres A. Pathophysiology of Pneumonia. Clinics in Chest Medicine. 2005;26:39-46.

[9] Sahn SA. Diagnosis and Management of Parapneumonic Effusions and Empyema. Clin Infect Dis. 2007;45:1480-1486. 
[10] Corcoran JP, Rahman NM. Effusions from infections: Parapneumonic pleural effusion and empyema. In: Light RW, Lee YCG, editors. Textbook of Pleural Diseases. 3rd ed. Boca Raton, Florida: CRC Press; 2016. p. 295-330.

[11] Kroegel C, Antony VB. Immunobiology of pleural inflammation: potential implications for pathogenesis, diagnosis and therapy. Eur. Respir. J. 1997;10:2411-2418.

[12] Broaddus VC, Boylan AM, Hoeffel JM, et al. Neutralization of IL-8 inhibits neutrophil influx in a rabbit model of endotoxin-induced pleurisy. J. Immunol. 1994;152:2960-2967.

[13] Broaddus VC, Hébert CA, Vitangcol RV, et al. Interleukin-8 is a major neutrophil chemotactic factor in pleural liquid of patients with empyema. Am. Rev. Respir. Dis. 1992;146:825-830.

[14] Light RW, Girard WM, Jenkinson SG, et al. Parapneumonic effusions. Am. J. Med. 1980;69:507-512.

[15] Idell S, Girard W, Koenig KB, et al. Abnormalities of pathways of fibrin turnover in the human pleural space. Am. Rev. Respir. Dis. 1991;144:187-194.

[16] Alemán C, Alegre J, Monasterio J, et al. Association between inflammatory mediators and the fibrinolysis system in infectious pleural effusions. Clin. Sci. 2003;105:601-607.

[17] Chung C-L, Hsiao S-H, Hsiao G, et al. Clinical importance of angiogenic cytokines, fibrinolytic activity and effusion size in parapneumonic effusions. PLoS ONE. 2013;8:e53169.

[18] Sahn SA, Reller LB, Taryle DA, et al. The contribution of leukocytes and bacteria to the low pH of empyema fluid. Am. Rev. Respir. Dis. 1983;128:811-815.

[19] Light RW, MacGregor MI, Ball WC, et al. Diagnostic significance of pleural fluid pH and PCO2. Chest. 1973;64:591-596.

[20] Mutsaers SE, Kalomenidis I, Wilson NA, et al. Growth factors in pleural fibrosis. Curr Opin Pulm Med. 2006;12:251-258.

[21] Sasse SA, Jadus MR, Kukes GD. Pleural fluid transforming growth factor-beta1 correlates with pleural fibrosis in experimental empyema. Am. J. Respir. Crit. Care Med. 2003;168:700-705.

[22] Kunz CR, Jadus MR, Kukes GD, et al. Intrapleural injection of transforming growth factor-beta antibody inhibits pleural fibrosis in empyema. Chest. 2004;126:1636-1644.

[23] Landreneau RJ, Keenan RJ, Hazelrigg SR, et al. Thoracoscopy for empyema and hemothorax. Chest. 1996;109:18-24.

[24] Neff CC, vanSonnenberg E, Lawson DW, et al. CT follow-up of empyemas: pleural peels resolve after percutaneous catheter drainage. Radiology. 1990;176:195-197.

[25] Hamm H, Light RW. Parapneumonic effusion and empyema. Eur. Respir. J. 1997;10:11501156.

[26] Lim WS, Lewis S, Macfarlane JT. Severity prediction rules in community acquired pneumonia: a validation study. Thorax. 2000;55:219-223. 
[27] Hasley PB, Albaum MN, Li YH, et al. Do pulmonary radiographic findings at presentation predict mortality in patients with community-acquired pneumonia? Arch. Intern. Med. 1996;156:2206-2212.

[28] Dean NC, Griffith PP, Sorensen JS, et al. Pleural Effusions at First ED Encounter Predict Worse Clinical Outcomes in Patients With Pneumonia. Chest. 2016;149:1509-1515.

[29] Corcoran JP, Wrightson JM, Belcher E, et al. Pleural infection: past, present, and future directions. The Lancet Respiratory Medicine. 2015;3:563-577.

[30] Tu C-Y, Chen C-H. Spontaneous bacterial empyema. Curr Opin Pulm Med. 2012;18:355-358.

[31] Smith JA, Mullerworth MH, Westlake GW, et al. Empyema thoracis: 14-year experience in a teaching center. Ann. Thorac. Surg. 1991;51:39-42.

[32] McCauley L, Dean N. Pneumonia and empyema: causal, casual or unknown. J Thorac Dis. 2015;7:992-998.

[33] Chalmers JD, Singanayagam A, Murray MP, et al. Risk factors for complicated parapneumonic effusion and empyema on presentation to hospital with community-acquired pneumonia. Thorax. 2009;64:592.

[34] Park C-K, Oh H-J, Choi H-Y, et al. Microbiological Characteristics and Predictive Factors for Mortality in Pleural Infection: A Single-Center Cohort Study in Korea. Trottein F, editor. PLOS ONE. 2016;11:e0161280.

[35] Maskell NA, Batt S, Hedley EL, et al. The Bacteriology of Pleural Infection by Genetic and Standard Methods and Its Mortality Significance. American Journal of Respiratory and Critical Care Medicine. 2006;174:817-823.

[36] Marks DJB, Fisk MD, Koo CY, et al. Thoracic Empyema: A 12-Year Study from a UK Tertiary Cardiothoracic Referral Centre. Wilkinson RJ, editor. PLoS ONE. 2012;7:e30074.

[37] Bedawi EO, Hassan M, Rahman NM. Recent developments in the management of pleural infection: a comprehensive review. The Clinical Respiratory Journal [Internet]. 2018 [cited 2018 Jul 14]; Available from: http://doi.wiley.com/10.1111/crj.12941.

[38] Corcoran JP, Wrightson JM, Belcher E, et al. Pleural infection: past, present, and future directions. The Lancet Respiratory Medicine. 2015;3:563-577.

[39] Davies HE, Davies RJO, Davies CWH, et al. Management of pleural infection in adults: British Thoracic Society pleural disease guideline 2010. Thorax. 2010;65:ii41-ii53.

[40] Lisboa T, Waterer GW, Lee YCG. Pleural infection: Changing bacteriology and its implications: Pleural infection: changing bacteriology. Respirology. 2011;16:598-603.

[41] Chen KY, Hsueh PR, Liaw YS, et al. A 10-year experience with bacteriology of acute thoracic empyema: emphasis on Klebsiella pneumoniae in patients with diabetes mellitus. Chest. 2000;117:1685-1689.

[42] Lin Y-C, Chen H-J, Liu Y-H, et al. A 30-month experience of thoracic empyema in a tertiary hospital: emphasis on differing bacteriology and outcome between the medical intensive care unit (MICU) and medical ward. South. Med. J. 2008;101:484-489. 
[43] Ip M, Osterberg LG, Chau PY, et al. Pulmonary melioidosis. Chest. 1995;108:1420-1424.

[44] Mukhopadhyay A. Bacteraemic melioidosis pneumonia: impact on outcome, clinical and radiological features. Journal of Infection. 2004;48:334-338.

[45] Hardie JM, Whiley RA. Classification and overview of the genera Streptococcus and Enterococcus. Soc. Appl. Bacteriol. Symp. Ser. 1997;26:1S-11S.

[46] Jerng JS, Hsueh PR, Teng $\amalg$, et al. Empyema thoracis and lung abscess caused by viridans streptococci. Am. J. Respir. Crit. Care Med. 1997;156:1508-1514.

[47] Boyanova L, Vladimir Djambazov, Gergova G, et al. Anaerobic microbiology in 198 cases of pleural empyema: a Bulgarian study. Anaerobe. 2004;10:261-267.

[48] Bhatnagar R, Maskell NA. Treatment of Complicated Pleural Effusions in 2013. Clinics in Chest Medicine. 2013;34:47-62.

[49] Blaschke AJ, Heyrend C, Byington CL, et al. Molecular analysis improves pathogen identification and epidemiologic study of pediatric parapneumonic empyema. Pediatr. Infect. Dis. J. 2011;30:289-294.

[50] Grau I, Ardanuy C, Calatayud L, et al. Invasive pneumococcal disease in healthy adults: increase of empyema associated with the clonal-type Sweden(1)-ST306. PLoS ONE. 2012;7:e42595.

[51] Xiol X, Castellví JM, Guardiola J, et al. Spontaneous bacterial empyema in cirrhotic patients: a prospective study. Hepatology. 1996;23:719-723.

[52] Ko SC, Chen KY, Hsueh PR, et al. Fungal empyema thoracis: an emerging clinical entity. Chest. 2000;117:1672-1678.

[53] Malhotra P, Aggarwal AN, Agarwal R, et al. Clinical characteristics and outcomes of empyema thoracis in 117 patients: A comparative analysis of tuberculous vs. non-tuberculous aetiologies. Respiratory Medicine. 2007;101:423-430.

[54] Lindstrom ST, Kolbe J. Community acquired parapneumonic thoracic empyema: predictors of outcome. Respirology. 1999;4:173-179.

[55] Okiror L, Coltart C, Bille A, et al. Thoracotomy and decortication: impact of culture-positive empyema on the outcome of surgery. European Journal of Cardio-Thoracic Surgery. 2014;46:901-906.

[56] Lee K-L, Chen W-L, Chen R-J, et al. Lipoteichoic acid upregulates plasminogen activator inhibitor-1 expression in parapneumonic effusions. Respirology. 2018;23:89-95.

[57] Alegre J, Jufresa J, Segura R, et al. Pleural-fluid myeloperoxidase in complicated and noncomplicated parapneumonic pleural effusions. Eur. Respir. J. 2002;19:320-325.

[58] Alemán C, Alegre J, Segura RM, et al. Polymorphonuclear elastase in the early diagnosis of complicated pyogenic pleural effusions. Respiration. 2003;70:462-467.

[59] Porcel JM, Vives M, Esquerda A. Tumor necrosis factor-alpha in pleural fluid: a marker of complicated parapneumonic effusions. Chest. 2004;125:160-164. 
[60] Iglesias D, Alegre J, Alemán C, et al. Metalloproteinases and tissue inhibitors of metalloproteinases in exudative pleural effusions. Eur. Respir. J. 2005;25:104-109.

[61] Porcel JM, Vives M, Cao G, et al. Biomarkers of infection for the differential diagnosis of pleural effusions. Eur. Respir. J. 2009;34:1383-1389.

[62] Dixon G, Lama-Lopez A, Bintcliffe OJ, et al. The role of serum procalcitonin in establishing the diagnosis and prognosis of pleural infection. Respir Res [Internet]. 2017 [cited 2018 Feb 13];18. Available from: https://www.ncbi.nlm.nih.gov/pmc/articles/PMC5291982/.

[63] Porcel JM, Valencia $\mathrm{H}$, Bielsa S. Factors influencing pleural drainage in parapneumonic effusions. Rev Clin Esp. 2016;216:361-366.

[64] Rovina N, Dima E, Psallidas I, et al. Interleukin-18 is up-regulated in infectious pleural effusions. Cytokine. 2013;63:166-171.

[65] Wu K-A, Wu C-C, Chen C-D, et al. Proteome profiling reveals novel biomarkers to identify complicated parapneumonic effusions. Scientific Reports. 2017;7:4026.

[66] Porcel JM, Esquerda A, Vives M, et al. Etiology of Pleural Effusions: Analysis of More Than 3,000 Consecutive Thoracenteses. Arch Bronconeumol. 2014;50:161-165.

[67] Wrightson JM, Wray JA, Street TL, et al. S113 Predictors Of Bacterial 'load' In Pleural Infection. Thorax. 2014;69:A60-A61.

[68] Menzies SM, Rahman NM, Wrightson JM, et al. Blood culture bottle culture of pleural fluid in pleural infection. Thorax. 2011;66:658-662.

[69] Maskell NA, Davies CWH, Nunn AJ, et al. U.K. Controlled Trial of Intrapleural Streptokinase for Pleural Infection. New England Journal of Medicine. 2005;352:865-874.

[70] Ruan S-Y, Chuang Y-C, Wang J-Y, et al. Revisiting tuberculous pleurisy: pleural fluid characteristics and diagnostic yield of mycobacterial culture in an endemic area. Thorax. 2012;67:822-827.

[71] Psallidas I, Kanellakis NI, Bhatnagar R, et al. A Pilot Feasibility Study in Establishing the Role of Ultrasound-Guided Pleural Biopsies in Pleural Infection (The AUDIO Study). Chest. 2018;

[72] Insa R, Marín M, Martín A, et al. Systematic use of universal 16S rRNA gene polymerase chain reaction (PCR) and sequencing for processing pleural effusions improves conventional culture techniques. Medicine (Baltimore). 2012;91:103-110.

[73] Taryle DA, Good JT, Morgan EJ, et al. Antibiotic concentrations in human parapneumonic effusions. J. Antimicrob. Chemother. 1981;7:171-177.

[74] Teixeira LR, Sasse SA, Villarino MA, et al. Antibiotic levels in empyemic pleural fluid. Chest. 2000;117:1734-1739.

[75] Hughes CE, Van Scoy RE. Antibiotic therapy of pleural empyema. Semin Respir Infect. 1991;6:94-102.

[76] Valcke Y, Pauwels R, Van der Straeten M. Pharmacokinetics of antibiotics in the lungs. Eur. Respir. J. 1990;3:715-722. 
[77] Davies HE, Davies RJO, Davies CWH. Management of pleural infection in adults: British Thoracic Society pleural disease guideline 2010. Thorax. 2010;65:ii41-ii53.

[78] Liapakis IE, Kottakis I, Tzatzarakis MN, et al. Penetration of newer quinolones in the empyema fluid. European Respiratory Journal. 2004;24:466-470.

[79] Saroglou M, Tryfon S, Ismailos G, et al. Pharmacokinetics of Linezolid and Ertapenem in experimental parapneumonic pleural effusion. Journal of Inflammation. 2010;7:22.

[80] Chatzika K, Manika K, Kontou P, et al. Moxifloxacin Pharmacokinetics and Pleural Fluid Penetration in Patients with Pleural Effusion. Antimicrob Agents Chemother. 2014;58:13151319.

[81] Shen KR, Bribriesco A, Crabtree T, et al. The American Association for Thoracic Surgery consensus guidelines for the management of empyema. J. Thorac. Cardiovasc. Surg. 2017;153:e129-e146.

[82] Shen KR, Bribriesco A, Crabtree T, et al. The American Association for Thoracic Surgery consensus guidelines for the management of empyema. The Journal of Thoracic and Cardiovascular Surgery. 2017;153:e129-e146.

[83] Birkenkamp K, O’Horo JC, Kashyap R, et al. Empyema management: A cohort study evaluating antimicrobial therapy. J. Infect. 2016;72:537-543.

[84] Ng T, Ryder BA, Maziak DE, et al. Treatment of postpneumonectomy empyema with debridement followed by continuous antibiotic irrigation. J. Am. Coll. Surg. 2008;206:11781183.

[85] Torbic H, Glasser N, Rostas SE, et al. Intrapleural Antimicrobial Irrigation for Postpneumonectomy Empyema in Patients With Lung Cancer. Journal of Pharmacy Practice. 2015;28:469-472.

[86] Rana MA, Rahman BAE, Mady AF, et al. Intra-Pleural Colistin Methanesulfonate Therapy for Pleural Infection caused by Carbapenem-Resistant Acinetobacter Baumannii: A Successful Case Report. Infect Dis Rep [Internet]. 2014 [cited 2018 Oct 10];6. Available from: https://www.ncbi.nlm.nih.gov/pmc/articles/PMC4178267/.

[87] Chao Y-K, Lee C-H, Liu K-S, et al. Sustained release of bactericidal concentrations of penicillin in the pleural space via an antibiotic-eluting pigtail catheter coated with electrospun nanofibers: results from in vivo and in vitro studies. Int J Nanomedicine. 2015;10:3329-3336.

[88] Lansley SM, Cheah HM, Varano Della Vergiliana JF, et al. Tissue plasminogen activator potently stimulates pleural effusion via a monocyte chemotactic protein-1-dependent mechanism. Am. J. Respir. Cell Mol. Biol. 2015;53:105-112.

[89] Tillett WS, Sherry S. THE EFFECT IN PATIENTS OF STREPTOCOCCAL FIBRINOLYSIN (STREPTOKINASE) AND STREPTOCOCCAL DESOXYRIBONUCLEASE ON FIBRINOUS, PURULENT, AND SANGUINOUS PLEURAL EXUDATIONS. J. Clin. Invest. 1949;28:173-190.

[90] Light RW, Nguyen T, Mulligan ME, et al. The In Vitro Efficacy of Varidase Versus Streptokinase or Urokinase for Liquefying Thick Purulent Exudative Material from Loculated Empyema. Lung. 2000;178:13-18. 
[91] Hall-Stoodley L, Nistico L, Sambanthamoorthy K, et al. Characterization of biofilm matrix, degradation by DNase treatment and evidence of capsule downregulation in Streptococcus pneumoniae clinical isolates. BMC Microbiol. 2008;8:173.

[92] Piccolo F, Popowicz N, Wong D, et al. Intrapleural tissue plasminogen activator and deoxyribonuclease therapy for pleural infection. J. 2015;7:999-1008.

[93] Florova G, Azghani A, Karandashova S, et al. Targeting of plasminogen activator inhibitor 1 improves fibrinolytic therapy for tetracycline-induced pleural injury in rabbits. Am. J. Respir. Cell Mol. Biol. 2015;52:429-437.

[94] Popowicz N, Idell S, Lee YCG. Pathogenesis of pleural infection: A complex warfare. Respirology. 2018;23:8-9.

[95] Lee YCG, Idell S, Stathopoulos GT. Translational Research in Pleural Infection and Beyond. Chest. 2016;150:1361-1370. 